\title{
Oleuropein attenuates the progression of heart failure in rats by antioxidant and antiinflammatory effects
}

\author{
Zeinab Janahmadi $^{1,2,3}$ • Ali Akbar Nekooeian ${ }^{1,2,4}$ • Ali Reza Moaref ${ }^{5,6}$ • \\ Masoomeh Emamghoreishi ${ }^{2}$
}

Received: 9 September 2016 / Accepted: 21 November 2016/Published online: 8 December 2016

(C) Springer-Verlag Berlin Heidelberg 2016

\begin{abstract}
Much of the beneficial effects of olive products have been attributed to oleuropein. This study examined the effects of oleuropein in rats with heart failure induced by permanent ligation of left coronary arteries. Twenty-four hours after the operation, the rats were assigned to five groups including a sham assigned to receive vehicle $(1 \mathrm{ml} /$ day $)$ and four coronary ligated groups assigned to receive vehicle or oleuropein at 5,10 , or $20 \mathrm{mg} / \mathrm{kg} / \mathrm{day}$. Five weeks later, echocardiographic and hemodynamic parameters, serum concentrations of oxidative stress, and inflammatory markers were determined. Myocardial infarction group receiving vehicle showed impaired hemodynamic and echocardiographic parameters as evidenced by decreased left ventricular systolic pressure, rate of rise and decrease of left ventricular pressure, stroke volume, ejection fraction, and cardiac output. In addition, significant reduction in superoxide dismutase and glutathione reductase was observed. Oleuropein treatment
\end{abstract}

Ali Akbar Nekooeian

nekooeiana@sums.ac.ir; nekooian@yahoo.com

1 Cardiovascular Pharmacology Research Lab, Department of Pharmacology, Medical School, Shiraz University of Medical Sciences, Shiraz 7134845794, Iran

2 Department of Pharmacology, Medical School, Shiraz University of Medical Sciences, Shiraz 7134845794, Iran

3 The Persian Gulf Marine Biotechnology Research Center, Bushehr University of Medical Sciences, Bushehr 7514763448, Iran

4 Department of Pharmacology, Medical School, Shiraz University of Medical Sciences, Zand Street, Shiraz, Fars 7134853185,, Iran

5 Department of Cardiology, Medical School, Shiraz University of Medical Sciences, Shiraz 7134845794, Iran

6 Cardiovascular Research Center, Shiraz University of Medical Sciences, Shiraz 7134845794, Iran prevented the reduction of these variables. Moreover, the group had a significantly higher infarct size and serum malondialdehyde, interleukin- $1 \beta$, and tumor necrosis factor- $\alpha$ than those of the sham group. Treatment with oleuropein prevented the increase of these variables. The results show that oleuropein attenuates the progression of heart failure, possibly by antioxidative and antiinflammatory effects.

Keywords Heart failure · Inflammatory cytokines · Oleuropein $\cdot$ Oxidative stress $\cdot$ Rat

\section{Introduction}

Heart failure, the inability to pump blood to meet the body's demand, is one of the most common causes of cardiovascular morbidity and mortality, and its prevalence is rapidly increasing (Gandhi et al. 2001; Sola et al. 2006). The most common cause of heart failure is myocardial infarction, which initiates a cascade of progressive structural and geometric changes in the left ventricle leading to progressive inability of the heart to maintain cardiac output.

Human and experimental models of heart failure are associated with hemodynamic and echocardiographic changes including progressive left ventricular dilation and increased left ventricular end-diastolic pressure (LVEDP) (Mercanoglu et al. 2010; Parveen et al. 2011; Radovanovic et al. 2012). They are also associated with decreased stroke volume (SV), fractional shortening (FS), ejection fraction (EF), cardiac output (CO), rate of rise of left ventricular pressure $(+\mathrm{dp} / \mathrm{dt})$, and rate of decrease of left ventricular pressure $(-\mathrm{dp} / \mathrm{dt})$ (Mercanoglu et al. 2010; Parveen et al. 2011; Radovanovic et al. 2012).

The pathogenesis and progression of heart failure have been attributed to increased reactive oxygen species 
(Giordano 2005) and lipid peroxidation markers like malondialdehyde (MDA). It has also been attributed to decreased antioxidant defenses like superoxide dismutase (SOD), catalase, and glutathione peroxidase (GPx) ( $\mathrm{Li}$ et al. 2012). Moreover, it has been related to increased proinflammatory cytokines such as interleukin-1 $\beta$ (IL$1 \beta$ ) and tumor necrosis factor- $\alpha$ (TNF- $\alpha$ ) (Ertl and Frantz 2005; Nian et al. 2004).

It has been reported that coronary heart disease, a major contributor to heart failure, is not a major indicator of mortality in men living in areas where Mediterranean diets constitute the major portions of their diets (Menotti et al. 1997). Olive products constitute a major portion of Mediterranean diet (Huang and Sumpio 2008). Much of the beneficial effects of Mediterranean diets have been attributed to polyphenol compounds (Andreadou et al. 2006; Huang and Sumpio 2008; Keys 1980; Manna et al. 2004). A strong correlation has been shown between the intake of foods rich in such compounds and low mortality due to coronary heart disease (Andreadou et al. 2006; Huang and Sumpio 2008; Keys 1980; Manna et al. 2004). Oleuropein, an important polyphenol of olive products, has been reported to have beneficial effects such as cardioprotective, antioxidant (Andreadou et al. 2006; Manna et al. 2004), antiinflammatory (Kim et al. 2010), antiplatelet (Omar 2010), and vasodilatory (Omar 2010). Moreover, we (Janahmadi et al., 2015) previously showed that pretreatment with oleuropein for 1 week prior to ligation of the coronary artery offered cardioprotection in the setting of acute myocardial infarction.

Given the beneficial effects of oleuropein, the present study was designed to examine the effects of oleuropein on the progression of rat model of heart failure induced by permanent ligation of the coronary artery. It also aimed to examine whether oleuropein's effects were mediated by antioxidant and antiinflammatory activities.

\section{Materials and methods}

\section{Animals}

Male Sprague-Dawley rats (200-250 g) were obtained from Laboratory Animal Breeding Centre, Shiraz University of Medical Sciences, Shiraz, Iran. They were maintained under standard conditions (12-h light/dark cycle at $20-24{ }^{\circ} \mathrm{C}$ and 25-35\% humidity) with standard rat chow and water ad libitum.

\section{Materials}

Oleuropein was purchased from Serva (Feinbiochemica, Heidelberg, Germany). Ketamine was obtained from Rotexmedica (Trittau, Germany) and xylazine from Alfasan
(Woerden, Holland). Thiobutabarbital (Inactin ${ }^{\circledR}$ ) was bought from ByK Gulden (Konstanz, Germany).

\section{Surgical procedures}

Animals were anesthetized with intraperitoneal injections of ketamine $(60 \mathrm{mg} / \mathrm{kg})$ and xylazine $(8 \mathrm{mg} / \mathrm{kg})$. They were then tracheally intubated and ventilated by a rodent respirator (Ugo Basile, Comerio, Italy) with room air at a frequency of 70 strokes/min and tidal volume of $1 \mathrm{ml} / 100 \mathrm{~g}$ body weight. Body temperature was maintained at $37 \pm 1{ }^{\circ} \mathrm{C}$ using a temperature controller (Physitemp Instruments, Clifton, USA) by means of a rectal probe. The chest cavity was opened at the level of left fourth intercostal space, and the hearts were exposed. The pericardial sacs were then opened, and left main coronary arteries were ligated at $2-4 \mathrm{~mm}$ from their origins using 5-0 prolene. In sham-operated rats, the sutures were passed around the coronary arteries but were not tightened. Afterwards, the chest walls and skin incisions were closed using absorbable and nonabsorbable suture materials, respectively (Janahmadi et al. 2015). The animals were then recovered from anesthesia and kept in single cages under standard conditions for 5 weeks, during which they were given daily vehicle ( $1 \mathrm{ml}$ distilled water), or oleuropein by oral gavage.

\section{Experimental design}

Starting from the next day after the operation, two groups including a sham-operated group assigned to receive vehicle (Sham-V) and a coronary artery-ligated group emerged. The coronary artery-ligated group was further randomly divided into four groups $(n=6-8)$ including a group assigned to receive vehicle (CAL-V) and three other groups assigned to receive oleuropein at $5 \mathrm{mg} / \mathrm{kg} / \mathrm{day}$ (CAL-Ole5), $10 \mathrm{mg} / \mathrm{kg} /$ day (CAL-Ole10), or $20 \mathrm{mg} / \mathrm{kg} /$ day (CAL-Ole20).

\section{Hemodynamic measurements}

After 5 weeks of treatment with vehicle of oleuropein, the animals were anesthetized with single intraperitoneal injections of thiobutabarbital $(100 \mathrm{mg} / \mathrm{kg})$. Heparinized salinefilled catheters were inserted into left carotid arteries for the measurement of systolic blood pressure (SBP), diastolic blood pressure (DBP), and heart rate (HR). Moreover, catheters were placed in right carotid arteries and advanced to left ventricles for the measurement of left ventricular systolic pressure (LVSP), LVEDP, and left ventricular $+\mathrm{dp} / \mathrm{dt}$ and $-\mathrm{dp} / \mathrm{dt}$. The animals were allowed to recover from surgical stress for $30 \mathrm{~min}$, and then, measurements of the hemodynamic variables were performed using a PowerLab data acquisition system (ML750, ADInstruments PowerLab System, Castle Hill, Australia). 


\section{Echocardiographic measurements}

After the measurement of hemodynamic variables, the animals were subjected to transthoracic echocardiography using a 10-5-MHz probe (Esaote, Florence, Italy). Two-dimensional guided M-mode images of the left ventricle were obtained from the short axis view at the level of papillary muscle. Left ventricular internal diameter in diastole (LVIDd) and left ventricular internal diameter in systole (LVIDs) were measured, and diastolic and diastolic volumes, SV, FS, EF, and $\mathrm{CO}$ were calculated (see in the following).

After the measurement of echocardiographic variables, blood samples were obtained and allowed to clot for $30 \mathrm{~min}$. The blood samples were then centrifuged at $1000 \mathrm{~g}$ for $20 \mathrm{~min}$, and their serums were separated and stored at $-80{ }^{\circ} \mathrm{C}$ until analysis.

\section{Assessment of infarct size}

Cardiac infarct size was assessed as previously described (Srikanth et al. 2009). The hearts were excised and washed in ice-cold $0.9 \%$ saline and were embedded into parafilm. After being kept at $-4{ }^{\circ} \mathrm{C}$ for $1 \mathrm{~h}$, they were cut transversely into 2-mm-thick slices. The slices were then incubated with $1 \%$ TTC at $37{ }^{\circ} \mathrm{C}$ for $25 \mathrm{~min}$. The stained slices were fixed with $10 \%$ formaldehyde overnight. The infarct sizes were determined via planimetry by using the NIH image software.

\section{Measurements of serum biomarkers}

Serum MDA (Bioassay Technology Laboratory, Shanghai, China), TNF- $\alpha$ (Glory Science, Del Rio, TX, USA), and IL$1 \beta$ (Boster Biological Technology, Wuhan, China) levels were measured by the ELISA method using the manufacturers' instructions. Serum SOD and glutathione reductase (GRx) were measured using chemical kits (Biorexfars, Shiraz, Iran).

\section{Calculations}

Mean arterial pressure (MAP) was calculated as DBP plus one third of arterial pulse pressure. Arterial resistance (AR) was calculated as MAP/CO. Diastolic and systolic volumes were calculated as $1.047[\mathrm{LVIDd}]^{3}$ and $1.047[\mathrm{LVIDs}]^{3}$, respectively. SV was calculated as diastolic volume - systolic volume. FS was calculated as [LVIDd - LVIDs] / LVIDd). EF was calculated as stroke volume / diastolic volume and $\mathrm{CO}$ as $\mathrm{SV} \times \mathrm{HR}$.

\section{Statistical analysis}

Data, presented as mean \pm SEM, were analyzed using oneway analysis of variance (ANOVA) followed by Duncan's multiple range test for pairwise comparisons. A $P$ value of $\leq 0.05$ was considered statistically significant. The data were analyzed using SigmaStat Statistical Software version 3.0 (San Jose, CA, USA). The illustrations were drawn using the SigmaPlot software (version 8.0) (San Jose, CA, USA).

\section{Results}

\section{Hemodynamic variables}

SBP, LVSP, $+\mathrm{dp} / \mathrm{dt}$, and $-\mathrm{dp} / \mathrm{dt}$ of the CAL-V group were significantly lower than those of the Sham-V group. However, AR and LVEDP of that group were significantly higher than those of the Sham-V group (Table 1).

There were no significant differences between the SBP, LVSP, $+\mathrm{dp} / \mathrm{dt}$, $-\mathrm{dp} / \mathrm{dt}$, or LVEDP of CAL-Ole5 and CAL-V groups. However, SBP, LVSP, +dp/dt, and $-\mathrm{dp} / \mathrm{dt}$ of CALOle10 and CAL-Ole20 groups were significantly higher than those of the CAL-V group, while AR and LVEDPs of such groups were significantly lower than those of the CAL-V group. There was no significant difference between the DBP, MAP, or HR of Sham-V, CAL-V, CAL-Ole5, CAL-Ole10, and CAL-Ole20 groups (Table 1).

\section{Echocardiographic variables}

The LVIDs, LVIDd, systolic volume, and diastolic volume of the CAL-V group were significantly higher than those of the Sham-V group. However, SV, EF, FS, and CO of the CAL-V group were significantly lower than those of the Sham-V group. There were no significant differences between the LVIDs, LVIDd, systolic and diastolic volumes, SV, EF, FS, and $\mathrm{CO}$ of CAL-Ole5 and CAL-V groups. The LVIDs, LVIDd, systolic volume, and diastolic volume of CALOle10 and CAL-Ole20 groups were significantly lower than those of the CAL-V group. However, the SV, EF, FS, and CO of CAL-Ole10 and CAL-Ole20 groups were significantly higher than those of the CAL-V group (Table 2 and Fig. 1).

\section{Infarct size}

There was no significant difference between the infarct size of CAL-Ole5 and CAL-V groups (Fig. 2). The infarct sizes of CAL-Ole10 and CAL-Ole20 groups were significantly lower than those of the CAL-V group.

\section{Serum biomarkers}

Serum levels of SOD and GRx of the CAL-V group were significantly lower than those of the Sham-V group (Fig. 3). There was no significant difference between the serum concentrations of SOD or GRx of CAL-V and CAL-Ole5 groups. 
Table 1 The values of hemodynamic parameters of all experimental groups

\begin{tabular}{|c|c|c|c|c|c|}
\hline & Sham-V & CAL-V & CAL-Ole5 & CAL-Ole10 & CAL-Ole20 \\
\hline $\mathrm{SBP}(\mathrm{mmHg})$ & $125.5 \pm 2.35$ & $110.8 \pm 2.4 \dagger$ & $116.1 \pm 2.9$ & $119.7 \pm 2.1 \ddagger$ & $122.4 \pm 2.4 \ddagger$ \\
\hline DBP (mmHg) & $93.4 \pm 2.5$ & $88.9 \pm 2.6$ & $95.5 \pm 2.7$ & $94.3 \pm 3.1$ & $98.9 \pm 2.4$ \\
\hline MAP (mmHg) & $104.3 \pm 1.9$ & $97.9 \pm 2.4$ & $102.1 \pm 2.5$ & $103.7 \pm 2.5$ & $106.2 \pm 2.2$ \\
\hline $\mathrm{AR}(\mathrm{mmHg} / \mathrm{ml})$ & $1.128 \pm 0.087$ & $1.579 \pm 0.077 \dagger$ & $1.268 \pm 0.037 \ddagger$ & $1.196 \pm 0.116+$ & $1.190 \pm 0.082 \ddagger$ \\
\hline HR (beats/min) & $411.9 \pm 8.2$ & $391.3 \pm 11.7$ & $396.1 \pm 10.1$ & $419.5 \pm 10.8$ & $399.2 \pm 11.4$ \\
\hline LVSP (mmHg) & $134.3 \pm 1.7$ & $111.8 \pm 2.7 \dagger$ & $117.3 \pm 2.6$ & $124.9 \pm 1.8+$ & $127.2 \pm 1.5 \$$ \\
\hline LVEDP (mmHg) & $-2.8 \pm 0.2$ & $4.5 \pm 0.4 \dagger$ & $3.7 \pm 0.3$ & $-0.2 \pm 0.3 \$$ & $-2.5 \pm 0.1 \ddagger$ \\
\hline$+\mathrm{dp} / \mathrm{dt}(\mathrm{mmHg} / \mathrm{s})$ & $5246 \pm 212$ & $3688 \pm 115 \dagger$ & $3803 \pm 119$ & $4474 \pm 104 \ddagger$ & $5285 \pm 87 t$ \\
\hline$-\mathrm{dp} / \mathrm{dt}(\mathrm{mmHg} / \mathrm{s})$ & $-4398 \pm 181$ & $-2952 \pm 81 \dagger$ & $-3204 \pm 76$ & $-3605 \pm 114 \ddagger$ & $-4153 \pm 86 t$ \\
\hline
\end{tabular}

Data are shown as mean \pm SEM, $n=6-8$ each group

$S B P$ systolic blood pressure, $D B P$ diastolic blood pressure, MAP mean arterial pressure, $A R$ arterial resistance, $H R$ heart rate, $L V S P$ left ventricular systolic pressure, $L V E D P$ left ventricular end-diastolic pressure, $+d p / d t$ rate of rise of left ventricular pressure, $-d p / d t$ rate of decrease of left ventricular pressure, Sham- $V$ sham group receiving vehicle (1 ml distilled water/day), $C A L-V$ coronary artery-ligated group receiving vehicle, $C A L-O l e 5$ coronary artery-ligated group receiving oleuropein at $5 \mathrm{mg} / \mathrm{kg} /$ day, $C A L$-Ole10 coronary artery-ligated group receiving oleuropein at $10 \mathrm{mg} / \mathrm{kg} / \mathrm{day}, \mathrm{CAL}-\mathrm{Ole} 20$ coronary artery-ligated group receiving oleuropein at $20 \mathrm{mg} / \mathrm{kg} /$ day

$\dagger$ Significant $(p \leq 0.05)$ difference from the Sham-V group; $₫$ Significant $(p \leq 0.05)$ difference from the CAL-V group

The serum concentrations of SOD and GRx of CAL-Ole10 and CAL-Ole20 groups were significantly higher than those of the CAL-V group (Fig. 3).

Serum level of MDA of the CAL-V group was significantly higher than that of the Sham- $\mathrm{V}$ group (Fig. 3). There was no significant difference between the serum concentrations of MDA of CAL-V and CAL-Ole5 groups. Serum MDA concentration of CAL-Ole10 and CAL-Ole20 groups was significantly lower than that of the CAL-V group (Fig. 3).

Serum levels of IL- $1 \beta$ and TNF- $\alpha$ of the CAL-V group were significantly higher than those of the Sham $-\mathrm{V}$ group (Fig. 4). There was no significant difference between serum concentrations of IL- $1 \beta$ or TNF- $\alpha$ of CAL-V and CAL-Ole 5 groups. Serum concentrations of IL-1 $\beta$ or TNF- $\alpha$ of CAL-Ole10 and CAL-Ole20 groups were significantly lower than those of the CAL-V group (Fig. 4).

\section{Discussion}

The findings of the present study show that permanent ligation of left main coronary results in heart failure and that oleuropein offers cardioprotection in rats with heart failure They also show that cardioprotection offered by oleuropein
Table 2 The values of echocardiographic parameters of all experimental groups

\begin{tabular}{lccccl}
\hline & \multicolumn{1}{l}{ Sham-V } & CAL-V & CAL-Ole5 & CAL-Ole10 & CAL-Ole20 \\
\hline LVIDs (cm) & $0.32 \pm 0.02$ & $0.80 \pm 0.03 \dagger$ & $0.79 \pm 0.04$ & $0.66 \pm 0.04 \ddagger$ & $0.69 \pm 0.02 \ddagger$ \\
LVIDd (cm) & $0.64 \pm 0.02$ & $0.87 \pm 0.02 \dagger$ & $0.89 \pm 0.03$ & $0.79 \pm 0.037$ & $0.82 \pm 0.02$ \\
SYS VOL (ml) & $0.036 \pm 0.01$ & $0.54 \pm 0.05 \dagger$ & $0.53 \pm 0.08$ & $0.32 \pm 0.06 \ddagger$ & $0.34 \pm 0.03 \ddagger$ \\
DIA VOL (ml) & $0.28 \pm 0.03$ & $0.70 \pm 0.05 \dagger$ & $0.75 \pm 0.08$ & $0.54 \pm 0.08$ & $0.58 \pm 0.03$ \\
SV (ml) & $0.24 \pm 0.02$ & $0.17 \pm 0.01 \dagger$ & $0.21 \pm 0.01$ & $0.22 \pm 0.02 \ddagger$ & $0.24 \pm 0.01 \ddagger$ \\
EF $(\%)$ & $87.1 \pm 1.9$ & $23.7 \pm 1.7 \dagger$ & $30.18 \pm 2.9$ & $42.3 \pm 3.1 \ddagger$ & $41.7 \pm 2.5 \ddagger$ \\
FS (\%) & $50.4 \pm 2.8$ & $8.7 \pm 0.7 \dagger$ & $11.4 \pm 1.2$ & $16.9 \pm 1.6 \ddagger$ & $16.4 \pm 1.1 \ddagger$ \\
CO (ml/min) & $95.7 \pm 7.5$ & $63.4 \pm 3.1 \dagger$ & $81.0 \pm 3.2$ & $91.1 \pm 9.6 \ddagger$ & $93.8 \pm 6.3 \ddagger$ \\
\hline
\end{tabular}

Data are shown as mean $\pm \mathrm{SEM}, n=6-8$ each group

LVIDs left ventricular internal diameter in systole, $L V I D d$ left ventricular internal diameter in diastole, SYS VOL systolic volume, DIA VOL diastolic volume, $S V$ stroke volume, $E F$ ejection fraction, $F S$ fractional shortening, $C O$ cardiac output, $S h a m-V$ sham-operated group receiving vehicle ( $1 \mathrm{ml}$ distilled water/day), $C A L-V$ coronary arteryligated group receiving vehicle, $C A L-O l e 5$ coronary artery-ligated group receiving oleuropein at $5 \mathrm{mg} / \mathrm{kg} / \mathrm{day}$, CAL-Ole10 coronary artery-ligated group receiving oleuropein at $10 \mathrm{mg} / \mathrm{kg} / \mathrm{day}, \mathrm{CAL}$-Ole20 coronary arteryligated group receiving oleuropein at $20 \mathrm{mg} / \mathrm{kg} /$ day

$\dagger$ Significant $(p \leq 0.05)$ difference from the Sham-V group; $\ddagger$ significant $(p \leq 0.05)$ difference from the CAL-V group 
Fig. 1 Representative echocardiography photographs. Two-dimensional, upper plane, and M-mode, lower plane, short-axis views of left ventricle at the level of papillary muscles of all experimental groups. Sham-V sham-operated group receiving vehicle (1 $\mathrm{ml}$ distilled water/day), $C A L-V$ coronary artery-ligated group receiving the vehicle, $C A L$ Ole10 coronary artery-ligated group receiving oleuropein at $10 \mathrm{mg} / \mathrm{kg} /$ day, CAL-Ole20 coronary arteryligated group receiving oleuropein at $20 \mathrm{mg} / \mathrm{kg} /$ day

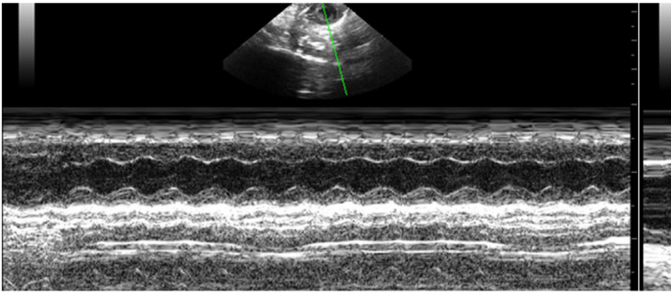

Sham-V

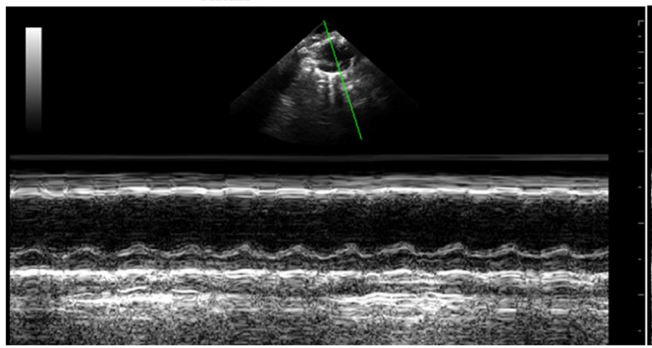

CAL-Ole10

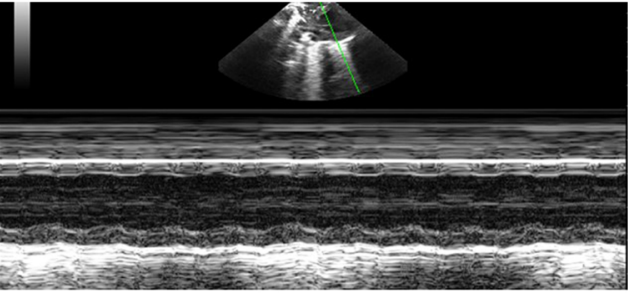

CAL-V

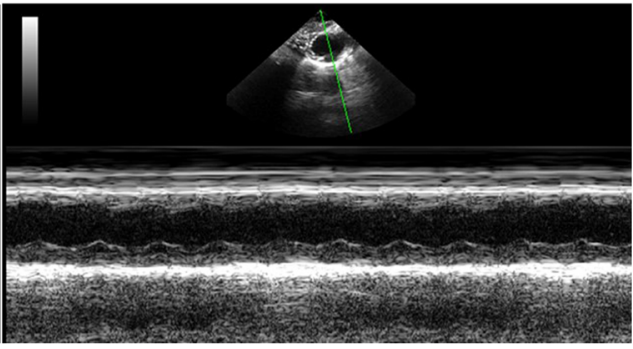

CAL-Ole20 might be related to the oleuropein's amelioration of oxidative stress and release of proinflammatory cytokines.

In a previous study (Janahmadi et al. 2015), we showed that oleuropein, given as pretreatment for 1 week prior to ligation of the coronary artery, was able to offer protection against acute myocardial infarction. The study showed that oleuropein cardioprotection might be mediated by reduction of oxidative stress and proinflammatory cytokines. The present study aimed to examine the treatment effects of oleuropein on the progression of heart failure. The hemodynamic and echocardiographic findings of the present study show that permanent ligation of the coronary artery for 5 weeks results in heart failure. Our study shows that cardiac hemodynamic parameters including LVSP, $+\mathrm{dp} / \mathrm{dt}$, and $-\mathrm{dp} / \mathrm{dt}$ were significantly lower, and LVEDP was higher in coronary artery-ligated group receiving vehicle than in sham-operated group. Such findings are similar to those of previous studies using the same model (Huang et al. 2009) or other models (Garjani et al. 2011) of experimental as well as human (Aurigemma et al. 2006) heart failure. Moreover, echocardiographic assessments showed that LVIDs, LVIDd, systolic volume, and diastolic volume of coronary artery-ligated rats receiving vehicle were significantly higher, while SV, EF, FS, and $\mathrm{CO}$ of that group were significantly lower than those of the sham-operated group. The echocardiographic findings are similar to those of earlier studies on experimental (Huang et al. 2009; Mercanoglu et al. 2010; Zhang et al. 2014; Zhou et al. 2007) and clinical (Radovanovic et al. 2012) heart failure.
Fig. 2 a Infarct size (as a percentage of the left ventricle) of coronary artery-ligated groups and b representative photographs of myocardial slices from shamoperated and coronary arteryligated groups. Sham- $V$ sham group receiving vehicle (1 $\mathrm{ml}$ distilled water/day), $C A L-V$ coronary arteryligated group receiving vehicle (1 $\mathrm{ml}$ distilled water/day), $C A L$ Ole- 5 coronary artery-ligated group receiving oleuropein at $5 \mathrm{mg} / \mathrm{kg}$ / day, CAL-Ole10 coronary arteryligated group receiving oleuropein at $10 \mathrm{mg} / \mathrm{kg} /$ day, CAL-Ole 20 coronary artery-ligated group receiving oleuropein at $20 \mathrm{mg} / \mathrm{kg} /$ day. Data are shown as mean \pm SEM, $n=6-8$ each group. $¥$ Significant difference $(p \leq 0.05)$ from CAL-V

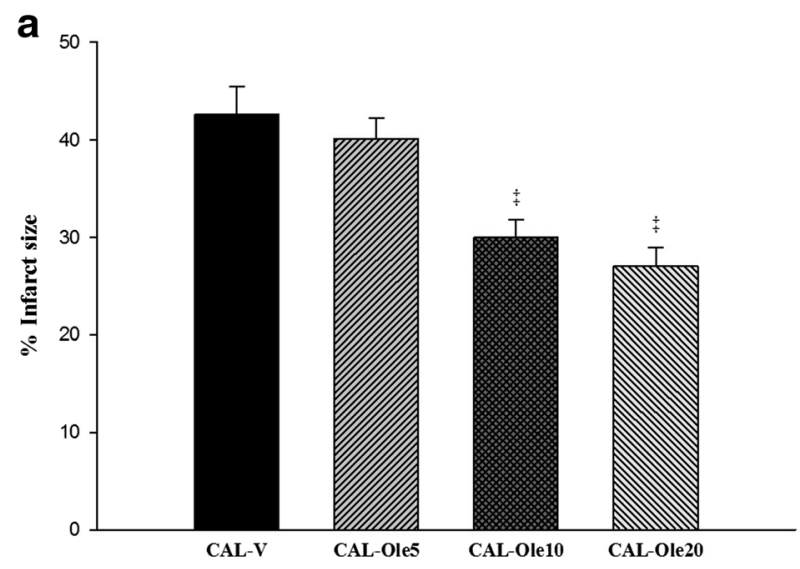

b
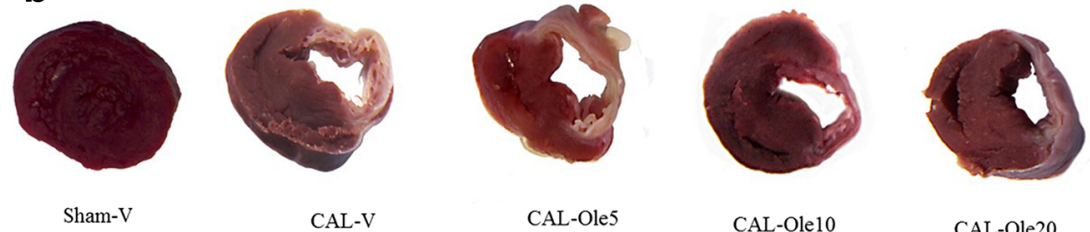

CAL-Ole20 
Fig. 3 Serum levels of biomarkers of oxidative stress of all experimental groups. Sham-V sham-operated group receiving vehicle ( $1 \mathrm{ml}$ distilled water/day), $C A L-V$ coronary artery-ligated group receiving vehicle, $C A L$ Ole-5 coronary artery-ligated group receiving oleuropein at $5 \mathrm{mg} / \mathrm{kg} /$ day, CAL-Ole10 coronary artery-ligated group receiving oleuropein at $10 \mathrm{mg} / \mathrm{kg}$ day, CAL-Ole 20 coronary arteryligated group receiving oleuropein at $20 \mathrm{mg} / \mathrm{kg} /$ day. Data are shown as mean \pm SEM, $n=6$ 8 each group. $\dagger$ Significant difference $(p \leq 0.05)$ from ShamV. $₫$ Significant difference $(p \leq 0.05)$ from CAL-V
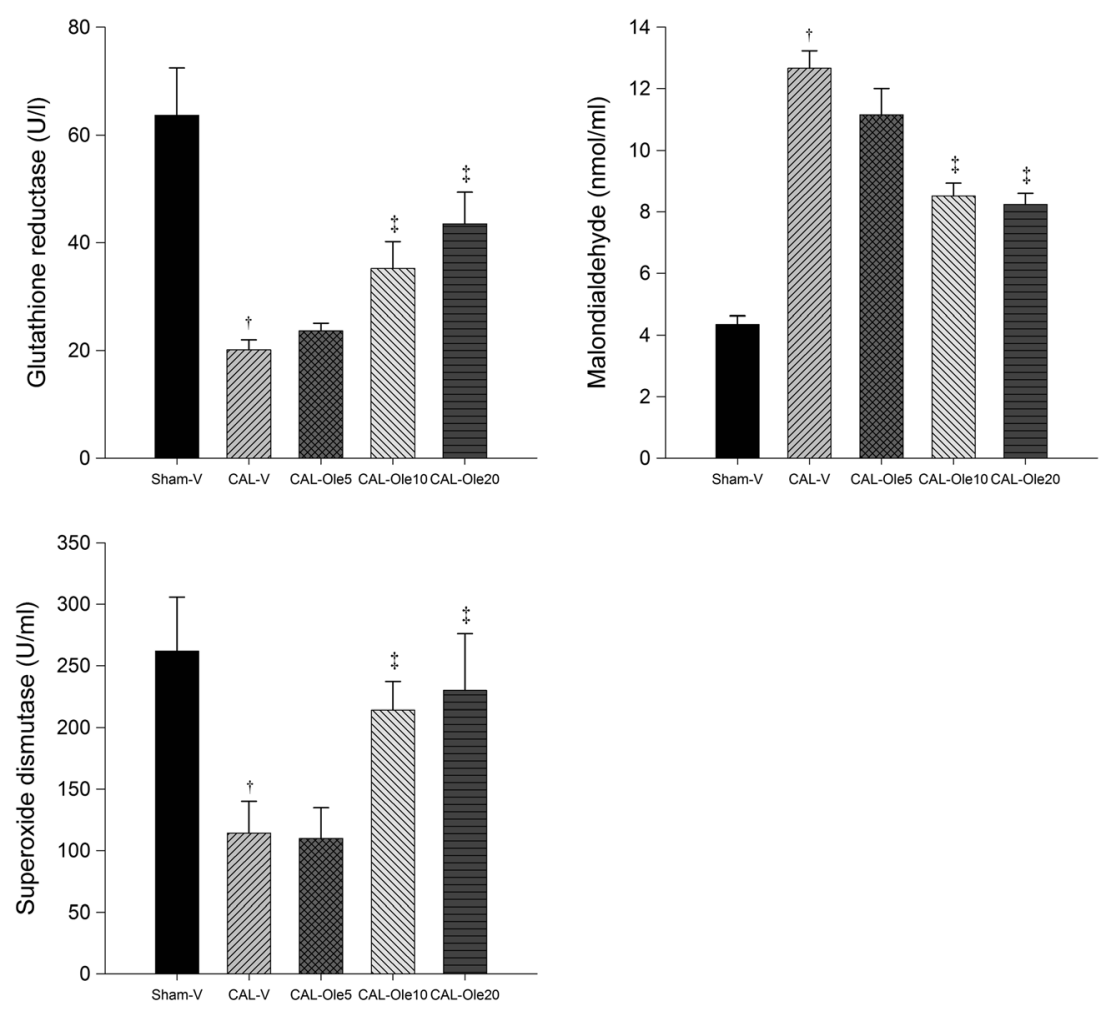

The progressive worsening of heart failure has been attributed to a number of mechanisms including the increase of oxidative stress (Giordano 2005), release of proinflammatory cytokines (Bozkurt et al. 2010), and neurohormonal activation (McAlpine et al. 1988). We, therefore, measured the serum concentrations of indices of oxidative stress such as SOD, GRx and MDA and proinflammatory cytokines such as IL$1 \beta$ and TNF- $\alpha$. Serum concentration of markers of oxidative stress and proinflammatory cytokines can predict the severity of heart failure as they were correlated with the severity of it (Wojciechowska et al. 2014; Zarrouk-Mahjoub et al. 2016). Our findings show that the present model of heart failure is

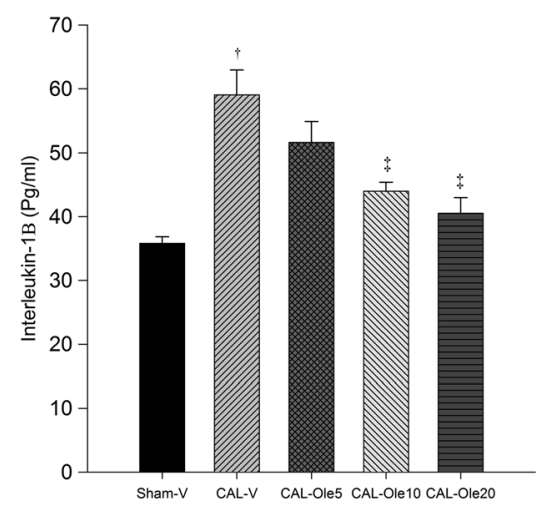

Fig. 4 Serum levels of proinflammatory cytokines of all experimental groups. Sham- $V$ sham-operated group receiving vehicle $(1 \mathrm{ml}$ distilled water/day), $C A L-V$ coronary artery-ligated group receiving vehicle, $C A L-O l e 5$ coronary artery-ligated group receiving oleuropein at $5 \mathrm{mg} / \mathrm{kg} /$ day, $C A L-O l e 10$ coronary artery-ligated group receiving associated with increased oxidative stress and release of proinflammatory cytokines. It has been suggested that oxidative stress via numerous mechanisms such as direct cytotoxic and negative inotropic (Ferrari et al. 1998), cytokine-stimulating (Mak and Newton 2001), and apoptotic (Kumar et al. 2002) effects contributes to the development of heart failure. Moreover, the contribution of proinflammatory cytokines to the development of the disease has been related to their ability to directly affect cardiac structure and contractile function (Hegewisch et al. 1990; Klug et al. 1993).

Oleuropein, at two largest doses of 10 and $20 \mathrm{mg} / \mathrm{kg} /$ day, attenuated the decrease of SBP, LVSP, $+\mathrm{dp} / \mathrm{dt}$, and $-\mathrm{dp} / \mathrm{dt}$ and

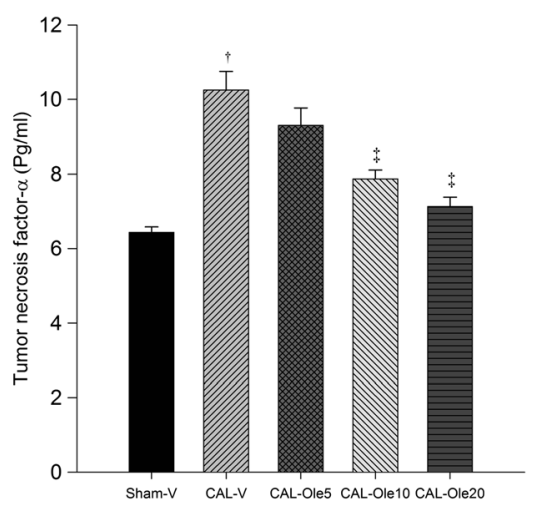

oleuropein at $10 \mathrm{mg} / \mathrm{kg} / \mathrm{day}, \mathrm{CAL}$-Ole20 coronary artery-ligated group receiving oleuropein at $20 \mathrm{mg} / \mathrm{kg} /$ day. Data are shown as mean $\pm \mathrm{SEM}$, $n=6-8$ each group. †Significant difference $(p \leq 0.05)$ from Sham-V. $\ddagger$ Significant difference $(p \leq 0.05)$ from CAL-V 
the increase of LVEDP and infarct size in coronary arteryligated rats. It also prevented the increase of LVIDs, LVIDd, systolic volume, and diastolic volume and the decrease of SV, $\mathrm{EF}, \mathrm{FS}$, and $\mathrm{CO}$ in such rats. These findings show that oleuropein at the two latter doses has cardioprotective effects. Such a conclusion receives support from previous studies demonstrating that oleuropein was cardioprotective against acute and chronic doxorubicin-induced cardiotoxicities (Andreadou et al. 2014; Andreadou et al. 2007) and ischemia-reperfusion injuries (Andreadou et al. 2006).

The cardioprotective effects of oleuropein have been attributed to several mechanisms such as reduction of oxidative and nitrosative stress (Andreadou et al. 2007) as well as antiplatelet (Petroni et al. 1995), hypolipidemic (Andreadou et al. 2006), and antiinflammatory (Andreadou et al. 2014) activities. We, therefore, sought to measure serum levels of SOD, GRx, MDA, IL- $1 \beta$, and TNF- $\alpha$ to examine the role of oxidative stress and proinflammatory cytokines in the oleuropein's cardioprotective effects. Our findings show that oleuropein reduces proinflammatory cytokines and increased antioxidant markers. Such findings are similar to those that reported that oleuropein reduced prooxidants and proinflammatory cytokines and increased antioxidant markers in adriamycin cardiotoxicity (Andreadou et al. 2014; Andreadou et al. 2007) and myocardial ischemia/reperfusion (Andreadou et al. 2006; Manna et al. 2004) studies.

Hemodynamically speaking, part of the beneficial effects of oleuropein might be related to its hypotensive and vasodilatory effects. We (Nekooeian et al. 2014) and others (Romero et al. 2016; Zarzuelo et al. 1991) showed that oleuropein possessed hypotensive and vasodilatory effects. It is interesting that, nonetheless, treatment with oleuropein was associated with increased SBP in the present model of heart failure, which was associated with reduced SBP and increased AR. The possible explanation for such an effect is that oleuropein, by virtue of its vasodilating activity, reduced AR and afterload. The reduced afterload may have helped that failing heart to function effectively and increase $\mathrm{CO}$ and consequently SBP. This explanation is in agreement with the most recent guidelines on the effectiveness of the use of vasodilators in the management of human heart failure (Yancy et al. 2013).

Taken together, our findings receive support from and give support to the widely accepted belief that Mediterranean diets, by virtue of richness in olive products, are associated with lower morbidity and mortality due to coronary heart diseases (Keys 1997). Given the role of coronary heart diseases in the initiation and progression of heart failure, our findings may be of clinical and practical relevance. Our findings may lay the ground for subsequent studies of oleuropein in other animal models of the disease and human heart failure and future use of the compound in the setting of human heart failure.
One limitation of the present study was the absence of a group of sham-operated rats receiving oleuropein diet. The presence of such a group could provide a chance to compare the effects of oleuropein in sham-operated and heart failure rats.

In conclusion, the findings of the present study indicate that oleuropein is cardioprotective in rats with heart failure, and such an effect might be mediated by antioxidant and antiinflammatory activities.

Acknowledgements This work was supported by a grant (91-6357) from the Vice Presidency for Research, Shiraz University of Medical Sciences.

Compliance with ethical standards All procedures were approved by the Institutional Committee for Ethics, Care, and Use of Animals.

Conflict of interest The authors declare that they have no conflict of interest.

\section{References}

Andreadou I et al (2006) The olive constituent oleuropein exhibits antiischemic, antioxidative, and hypolipidemic effects in anesthetized rabbits. J Nutr 136:2213-2219

Andreadou I et al (2007) Acute doxorubicin cardiotoxicity is successfully treated with the phytochemical oleuropein through suppression of oxidative and nitrosative stress. J Mol Cell Cardiol 42:549-558. doi:10.1016/j.yjmcc.2006.11.016

Andreadou I et al (2014) Oleuropein prevents doxorubicin-induced cardiomyopathy interfering with signaling molecules and cardiomyocyte metabolism. J Mol Cell Cardiol 69:4-16. doi:10.1016/j. yjmcc.2014.01.007

Aurigemma GP, Zile MR, Gaasch WH (2006) Contractile behavior of the left ventricle in diastolic heart failure: with emphasis on regional systolic function. Circulation 113:296-304. doi:10.1161/CIRCULATIONAHA.104.481465

Bozkurt B, Mann DL, Deswal A (2010) Biomarkers of inflammation in heart failure. Heart Fail Rev 15:331-341. doi:10.1007/s10741-0099140-3

Ertl G, Frantz S (2005) Healing after myocardial infarction. Cardiovasc Res 66:22-32. doi:10.1016/j.cardiores.2005.01.011

Ferrari R et al (1998) Oxidative stress during myocardial ischaemia and heart failure. Eur Heart J 19(Suppl B):B2-11

Gandhi SK, Powers JC, Nomeir AM, Fowle K, Kitzman DW, Rankin KM, Little WC (2001) The pathogenesis of acute pulmonary edema associated with hypertension. N Eng1 J Med 344:17-22. doi:10.1056/NEJM200101043440103

Garjani A, Andalib S, Biabani S, Soraya H, Doustar Y, Maleki-Dizaji N (2011) Combined atorvastatin and coenzyme Q10 improve the left ventricular function in isoproterenol-induced heart failure in rat. Eur J Pharmacol 666:135-141. doi:10.1016/j.ejphar.2011.04.061

Giordano FJ (2005) Oxygen, oxidative stress, hypoxia, and heart failure. J Clin Invest 115:500-508. doi:10.1172/JCI24408

Hegewisch S, Weh HJ, Hossfeld DK (1990) TNF-induced cardiomyopathy. Lancet 335:294-295

Huang CL, Sumpio BE (2008) Olive oil, the mediterranean diet, and cardiovascular health. J Am Coll Surg 207:407-416. doi:10.1016/j.jamcollsurg.2008.02.018 
Huang CX et al (2009) Ghrelin inhibits post-infarct myocardial remodeling and improves cardiac function through anti-inflammation effect. Peptides 30:2286-2291. doi:10.1016/j.peptides.2009.09.004

Janahmadi Z, Nekooeian AA, Moaref AR, Emamghoreishi M (2015) Oleuropein offers cardioprotection in rats with acute myocardial infarction. Cardiovascular toxicology 15:61-68. doi:10.1007/s12012-0149271-1

Keys A (1980) Seven countries : a multivariate analysis of death and coronary heart disease / Ancel Keys, with Christ Aravanis [et al.]. Harvard University Press, Cambridge, Mass

Keys A (1997) Coronary heart disease in seven countries. 1970. Nutrition 13:250-252 discussion 249, 253

Kim Y, Choi Y, Park T (2010) Hepatoprotective effect of oleuropein in mice: mechanisms uncovered by gene expression profiling. Biotechnol J 5:950-960. doi:10.1002/biot.201000068

Klug D, Robert V, Swynghedauw B (1993) Role of mechanical and hormonal factors in cardiac remodeling and the biologic limits of myocardial adaptation. Am J Cardiol 71:46A-54A

Kumar D, Lou H, Singal PK (2002) Oxidative stress and apoptosis in heart dysfunction. Herz 27:662-668. doi:10.1007/s00059-0022430-3

Li C, Gao Y, Tian J, Xing Y, Zhu H, Shen J (2012) Long-term oral Asperosaponin VI attenuates cardiac dysfunction, myocardial fibrosis in a rat model of chronic myocardial infarction. Food Chem Toxicol 50:1432-1438. doi:10.1016/j.fct.2012.01.024

Mak S, Newton GE (2001) The oxidative stress hypothesis of congestive heart failure: radical thoughts. Chest 120:2035-2046

Manna C, Migliardi V, Golino P, Scognamiglio A, Galletti P, Chiariello M, Zappia V (2004) Oleuropein prevents oxidative myocardial injury induced by ischemia and reperfusion. J Nutr Biochem 15:461466. doi:10.1016/j.jnutbio.2003.12.010

McAlpine HM, Morton JJ, Leckie B, Rumley A, Gillen G, Dargie HJ (1988) Neuroendocrine activation after acute myocardial infarction. Br Heart J 60:117-124

Menotti A et al (1997) Changes in population cholesterol levels and coronary heart disease deaths in seven countries. Eur Heart J 18: $566-571$

Mercanoglu GO, Pamukcu B, Safran N, Mercanoglu F, Fici F, Gungor M (2010) Nebivolol prevents remodeling in a rat myocardial infarction model: an echocardiographic study. Anadolu Kardiyol Derg 10:18-27

Nekooeian AA, Khalili A, Khosravi MB (2014) Effects of oleuropein in rats with simultaneous type 2 diabetes and renal hypertension: a study of antihypertensive mechanisms. J Asian Nat Prod Res 16: 953-962. doi:10.1080/10286020.2014.924510

Nian M, Lee P, Khaper N, Liu P (2004) Inflammatory cytokines and postmyocardial infarction remodeling. Circ Res 94:1543-1553. doi:10.1161/01.RES.0000130526.20854.fa
Omar SH (2010) Oleuropein in olive and its pharmacological effects. Sci Pharm 78:133-154. doi:10.3797/scipharm.0912-18

Parveen A, Babbar R, Agarwal S, Kotwani A, Fahim M (2011) Mechanistic clues in the cardioprotective effect of Terminalia arjuna bark extract in isoproterenol-induced chronic heart failure in rats. Cardiovasc Toxicol 11:48-57. doi:10.1007/s12012-010-9099-2

Petroni A, Blasevich M, Salami M, Papini N, Montedoro GF, Galli C (1995) Inhibition of platelet aggregation and eicosanoid production by phenolic components of olive oil. Thromb Res 78:151-160

Radovanovic S et al (2012) Markers of oxidative damage and antioxidant enzyme activities as predictors of morbidity and mortality in patients with chronic heart failure. J Card Fail 18:493-501. doi:10.1016/j. cardfail.2012.04.003

Romero $\mathrm{M}$ et al (2016) Antihypertensive effects of oleuropein-enriched olive leaf extract in spontaneously hypertensive rats. Food Funct 7: 584-593. doi:10.1039/c5fo01101a

Sola S, Mir MQ, Lerakis S, Tandon N, Khan BV (2006) Atorvastatin improves left ventricular systolic function and serum markers of inflammation in nonischemic heart failure. J Am Coll Cardiol 47: 332-337. doi:10.1016/j.jacc.2005.06.088

Srikanth G, Prakash P, Tripathy N, Dikshit M, Nityanand S (2009) Establishment of a rat model of myocardial infarction with a high survival rate: a suitable model for evaluation of efficacy of stem cell therapy. J Stem Cells Regen Med 5:30-36

Wojciechowska C, Romuk E, Tomasik A, Skrzep-Poloczek B, Nowalany-Kozielska E, Birkner E, Jachec W (2014) Oxidative stress markers and C-reactive protein are related to severity of heart failure in patients with dilated cardiomyopathy. Mediat Inflamm 2014:147040. doi:10.1155/2014/147040

Yancy CW et al (2013) 2013 ACCF/AHA guideline for the management of heart failure: a report of the American College of Cardiology Foundation/American Heart Association Task Force on Practice Guidelines. J Am Coll Cardiol 62:e147-e239. doi:10.1016/j. jacc.2013.05.019

Zarrouk-Mahjoub S et al (2016) Pro- and anti-inflammatory cytokines in post-infarction left ventricular remodeling. Int J Cardiol 221:632 636. doi:10.1016/j.ijcard.2016.07.073

Zarzuelo A, Duarte J, Jimenez J, Gonzalez M, Utrilla MP (1991) Vasodilator effect of olive leaf. Planta Med 57:417-419. doi:10.1055/s-2006-960138

Zhang YJ et al (2014) Berberine attenuates adverse left ventricular remodeling and cardiac dysfunction after acute myocardial infarction in rats: role of autophagy. Clin Exp Pharmacol Physiol 41:9951002. doi:10.1111/1440-1681.12309

Zhou F, Hu SJ, Mu Y (2007) Protection effect of Wenxin Keli on isoproterenol induced heart failure in rats. Zhongguo Zhong Yao Za Zhi $32: 1676-1679$ 